\title{
Helium reflectivity and Debye temperature of graphene grown epitaxially on $\operatorname{Ru}(0001)$
}

\author{
A. Politano, ${ }^{1,2}$ B. Borca, ${ }^{1}$ M. Minniti, ${ }^{1}$ J. J. Hinarejos, ${ }^{1,3}$ A. L. Vázquez de Parga, ${ }^{1,2,3}$ D. Farías, ${ }^{1,3, *}$ and R. Miranda ${ }^{1,2,3}$ \\ ${ }^{1}$ Departamento de Física de la Materia Condensada, Universidad Autónoma de Madrid, Cantoblanco, E-28049 Madrid, Spain \\ ${ }^{2}$ Instituto Madrileño de Estudios Avanzados en Nanociencia (IMDEA-Nanociencia), Cantoblanco, E-28049 Madrid, Spain \\ ${ }^{3}$ Instituto de Ciencia de Materiales “Nicolás Cabrera”, Universidad Autónoma de Madrid, Cantoblanco, E-28049 Madrid, Spain
}

(Received 1 March 2011; revised manuscript received 14 June 2011; published 29 July 2011)

\begin{abstract}
It is shown that the surface of an epitaxial graphene monolayer grown on $\mathrm{Ru}(0001)$ could be used as a quite efficient external mirror for He-atom microscopy, with a specular reflectivity of $20 \%$ of the incident beam. Furthermore, the system is stable up to $1150 \mathrm{~K}$, and the He reflectivity remains almost unchanged after exposure to air. Additionally, the high reflectivity for $\mathrm{H}_{2}$ molecules (11\%) opens up the development of a $\mathrm{H}_{2}$ microprobe suitable for lithography. The Debye temperature for this epitaxial graphene monolayer has been determined from a study of the temperature dependence of the He specular intensity as a function of incident parameters. A value of $1045 \mathrm{~K}$ has been obtained, which is much higher than the $590 \mathrm{~K}$ reported for graphite under similar conditions, and close to the value of $1287 \mathrm{~K}$ calculated for isolated graphene.
\end{abstract}

DOI: 10.1103/PhysRevB.84.035450

PACS number(s): 68.65.Pq, 68.49.Bc, 34.35.+a

\section{INTRODUCTION}

Much effort has been devoted in recent years to the development of a scanning helium-atom microscope (SHeAM), ${ }^{1-4}$ in which a focused beam of neutral He atoms is used as imaging probe. In fact, the first micrograph using helium atoms as an imaging probe has been reported recently. ${ }^{2}$ The development of a SHeAM would imply a substantial improvement of our present knowledge of delicate biological materials, weak polymers, ceramics, and insulating glass surfaces, among other materials. Such samples are difficult to examine by electron microscopy techniques, due to sample charging or electron excitation effects. Likewise, scanning tunneling microscopy (STM) cannot be used, as it works only on conductive substrates. Atomic force microscopy could in principle be employed on these surfaces, but the complex tip-sample interaction often jeopardizes the interpretation of images.

On the other hand, and due to the low energies employed ( 10-100 meV), neutral helium atoms probe the topmost surface layer of any material in an absolutely nondestructive manner. ${ }^{5}$ Electron microscopes and x-ray microscopes as well as the recently developed helium-ion microscope ${ }^{6,7}$ all operate with beam energies in the $\mathrm{keV}$ range. In contrast, the low energy of the impinging neutral He atoms employed in SHeAM would avoid the appearance of unwanted beam-induced effects, including sputtering and the excitation of many surface modes like single-particle excitations and plasmons.

Currently, the main limitation for developing SHeAM is related to the focusing of atomic beams. Two different solutions are possible. The first one is the use of Fresnel zone plates, ${ }^{8,9}$ which presents the disadvantage of chromatic aberration. The most promising method is to use the He beam specularly reflected from an electrostatically bent single-crystal surface. ${ }^{1}$ Bent-crystal mirrors offer both achromatic focusing and large numerical apertures, whereby a high reflectivity is required for practical applications. ${ }^{10}$ Present prototypes of the SHeAM use a hydrogen-passivated $\mathrm{Si}(111)-(1 \times 1)$ mirror, which exhibits a reflectivity of $\sim 1 \%$ for He atoms. ${ }^{11}$ Such a low intensity of the focused beam limits the present lateral resolution of the SHeAM to about $230 \mu \mathrm{m},{ }^{12}$ although in principle it could be reduced to $50 \mathrm{~nm}$ if a larger beam intensity were available. Therefore, the realization of a more efficient mirror for SHeAM has hitherto been a grand challenge. In general, the main limitation to a high reflectivity is due to the large sensitivity of He atoms to even a small density of surface defects. ${ }^{5}$ In addition, the mirror for the SHeAM should be inert and thermally stable. As an example, despite the high reflectivity of the clean $\mathrm{Ru}(0001)$ surface, it is not suitable as a mirror, because its reflectivity is reduced to zero after exposure to reactive species. A clear advantage of inert mirrors is that they could be produced ex situ and delivered to the place were the SHeAM is located. We have also shown that quantum-stabilized, ultraflat, and ultrathin films of $\mathrm{Pb}$ might reflect substantially better than existing mirrors. ${ }^{13}$ The films, however, were not stable at $300 \mathrm{~K}$. More recently, we showed that the $\mathrm{Pb}(\sqrt{3} \times \sqrt{3}) R 30^{\circ} \mathrm{Si}(111)$ surface presents a specular He reflectivity of $15 \%$, and is stable up to $450 \mathrm{~K} .{ }^{14}$ However, this surface is destroyed by contamination when exposed to air.

Recent work has shown that a monolayer of graphene (MLG) can be grown with excellent crystalline quality on metal surfaces. ${ }^{15-18}$ The preparation of highly ordered graphene can be extended even up to the millimeter scale for epitaxial graphene grown on $\mathrm{Ru}(0001) .{ }^{19}$ No bond breakage was revealed even over the substrate steps. Furthermore, MLG can be grown also on thin films of (0001)-oriented $\mathrm{Ru}$ deposited on $\mathrm{SiO}_{2} / \mathrm{Si}$ (Ref. 20) and sapphire (0001) (Ref. 21) substrates, so as to allow the practical design of a bent-crystal mirror. Hence, MLG on Ru(0001) could be a good candidate for the construction of a mirror for focusing beams of neutral atoms and molecules, if the reflectivity and stability are appropriate.

In addition, the unusual electronic and optical properties of supported graphene have attracted widespread interest. ${ }^{22}$ In particular, understanding the atomic dynamics of graphene is interesting in view of the high thermal conductivity of graphene. The simplest view of the dynamics of atoms in twodimensional systems is given by the Debye temperature, ${ }^{23-25}$ which is related to the thermal vibration of the surface atoms. It provides important information about the thermal stability and hardness of materials, as well as about the strength of the bonds 
involved in the surface termination. Atomic beam scattering at thermal energies $(\sim 10-100 \mathrm{meV})$ provides a reliable way to obtain the surface Debye temperature of any material, since the scattering process is restricted to the outermost surface layer. $^{5}$

In this paper, we show that the surface of an epitaxial graphene monolayer grown on $\mathrm{Ru}(0001)$ has a large $\mathrm{He}$ specular reflectivity of $20 \%$ of the incident beam, is stable up to $1150 \mathrm{~K}$, and is inert after exposure to air. In addition, the high reflectivity obtained for $\mathrm{H}_{2}$ beams $(11 \%)$ opens up the development of a $\mathrm{H}_{2}$ microprobe suitable for lithography and molecular interferometry. Finally, the Debye temperature of the graphene monolayer on $\mathrm{Ru}(0001)$ has been found to be $1045 \mathrm{~K}$, which is much higher than the one reported for graphite, ${ }^{26}$ but surprisingly close to the value of $1287 \mathrm{~K}$ recently calculated for out-of-plane vibrations in isolated graphene. ${ }^{34}$ Possible reasons for this behavior are discussed.

\section{EXPERIMENTAL DETAILS}

Experiments have been carried out in two different ultrahigh-vacuum (UHV) chambers with base pressures in the low $10^{-10}$ mbar range. The first one is a helium-atom scattering apparatus described elsewhere. ${ }^{27}$ Briefly, the angular distribution of the scattered $\mathrm{He}$ atoms can be analyzed with a quadrupole mass spectrometer mounted on a twoaxis goniometer. The intensity of the incident beam can also be measured and used to normalize scattered beam intensities, thereby yielding absolute diffraction intensities. Since the incident and specular beams have similar widths, this normalization can be made just by comparing the intensities of the incident and specular peaks for given incidence conditions. The sample temperature was measured with a type-C thermocouple spot-welded to the sample. The second chamber contains a variable-temperature STM, employed to check the structural quality of the graphene monolayer.

Atomically clean, bulk-C-depleted, crystalline $\mathrm{Ru}(0001)$ surfaces were prepared by standard sputtering-annealing cycles followed by oxygen exposure at $1150 \mathrm{~K}$ and a final flash to $1500 \mathrm{~K}$. In both chambers, surface cleanliness and order were also checked using Auger electron spectroscopy and low-energy electron diffraction measurements, respectively. Graphene was obtained by dosing $\mathrm{C}_{2} \mathrm{H}_{4}$ (ethylene) onto the clean $\mathrm{Ru}(0001)$ substrate at $1150 \mathrm{~K}$. The high temperature of the sample during deposition favors increase of the size of monolayer graphene islands ${ }^{28}$ and, moreover, allows the substrate to be kept clean so as to avoid any contaminantinduced effect on graphene growth. The completion of the first layer was reached upon an exposure to $3 \times 10^{-8} \mathrm{mbar}$ ethylene for $10 \mathrm{~min}$ [a dose equivalent to 24 langmuirs (L)]. The temperature was then held at $1150 \mathrm{~K}$ for a further $60 \mathrm{~s}$ after removing the $\mathrm{C}_{2} \mathrm{H}_{4}$ gas from the chamber. Such optimal conditions were determined from a combined STM and Helium Atom Scattering (HAS) analysis of graphene growth on $\mathrm{Ru}(0001)$. The air exposure was performed by removing the sample from the UHV chamber through a fast load-lock entry and reintroducing it into the UHV chamber for HAS and STM measurements. Oxygen was dosed through a leak valve.

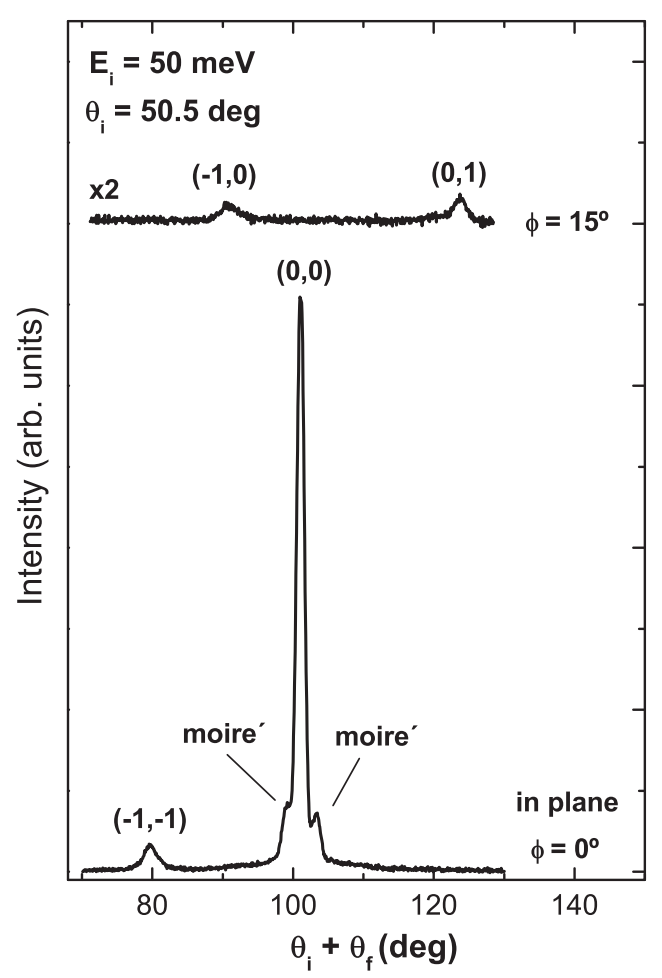

FIG. 1. Angular distribution of a beam of $\mathrm{He}$ atoms with an incident energy of $50 \mathrm{meV}$ scattered from the $\mathrm{MLG} / \mathrm{Ru}(0001)$ surface along the [11 20$]$ azimuth. The incident angle is $\Theta_{i}=50.5^{\circ}$. Diffraction peaks corresponding to the graphene overlayer can be seen in both the in-plane and the out-of-plane spectra. The sample temperature is $100 \mathrm{~K}$.

\section{RESULTS AND DISCUSSION}

Specular scattering dominates the He diffraction pattern of MLG/Ru(0001), as can be seen in the diffraction spectra shown in Fig. 1. The existence of a low-intensity but wellresolved ( $\overline{1} \overline{1})$ diffraction peak is the fingerprint of the existence of a well-ordered MLG, also confirmed by STM images (see below). Other weak diffraction features, assigned to the (10) and (01) peaks of MLG, are present in the spectra recorded at $15^{\circ}$ out of plane. The in-plane spectrum also shows the firstorder peaks corresponding to the moiré superstructure closer to the specular peak. Note the low total intensity of diffracted peaks as compared to that of the specular peak, which is a consequence of the low corrugation of $\mathrm{MLG} / \mathrm{Ru}(0001)$, estimated as $\sim 0.15-0.30 \AA .^{29}$

A comparison of the incident He beam profile with the one corresponding to the specular peak recorded at $E_{i}=32 \mathrm{meV}$ and $\Theta_{i}=50^{\circ}$ is shown in Fig. 2. The specular absolute reflectivity is $20 \%$, which is a factor of $\sim 20$ times higher than for the H-passivated $\mathrm{Si}(111)$ surface under similar scattering conditions. ${ }^{11}$ Likewise, an $11 \%$ reflectivity was obtained for a $\mathrm{H}_{2}$ beam scattered off $\mathrm{MLG} / \mathrm{Ru}(0001)$, whereas to date the highest $\mathrm{H}_{2}$ reflectivity reported for $\mathrm{H}_{2}$ beams was $2.6 \%$ in the case of $\mathrm{Si}(111)-\mathrm{H}(1 \times 1) .{ }^{11}$ An intense and focused beam of hydrogen might be a promising tool for lithography in wafer-scale fabrication of molecular junctions. ${ }^{30}$

Helium-atom scattering at thermal energies provides a reliable way to obtain the surface Debye temperature, since the 


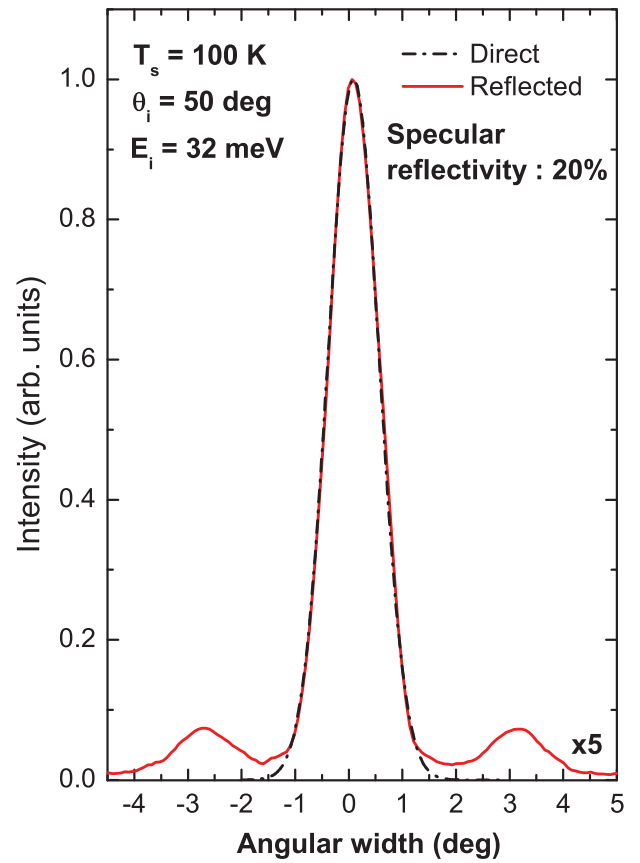

FIG. 2. (Color online) Comparison of the incident $\mathrm{He}$ beam intensity with the specular $\mathrm{He}$ reflectivity for MLG/Ru(0001). The incident energy is $32 \mathrm{meV}$.

scattering process occurs at $\sim 3 \AA$ away from the topmost atom cores of the solids surface. Both zero-point motion and thermal vibrations of the surface atoms lead to inelastic scattering of the incoming atoms. The main observable effect of this is a thermal attenuation of the coherent diffraction intensities, without a change of the peak shape. ${ }^{31}$ Note also that the Debye temperature values determined using other probes could in principle be slightly different from the one obtained in our experiments.

The thermal attenuation of the specular beam for scattering of He atoms from MLG is shown in Fig. 3 as a function of surface temperature, incident beam energy, and angle of incidence. Note the exponential attenuation of the specular intensity. The exponential temperature dependence was verified for two different angles of incidence $\left(\Theta_{i}=45^{\circ}\right.$ and $\left.60^{\circ}\right)$ and three different beam energies. For comparison, Fig. 3 also includes the He specular attenuation curve reported in Ref. 26 for highly oriented pyrolytic graphite (HOPG) (which has been rescaled). Again an exponential dependence is observed, but the slope is much larger for HOPG than for MLG/Ru(0001) under almost the same incident conditions (compare the data points for $E_{i}=64 \mathrm{meV}$ and $\Theta_{i}=45^{\circ}$ ), which indicates a smaller value of the corresponding Debye temperature.

The intensity $I(T)$ of a diffraction peak at finite temperature $T$ is related to the intensity $I_{0}$ from the lattice at rest by

$$
I(T)=I_{0} e^{-2 W(T)},
$$

where $\exp [-2 W(T)]$ is the Debye-Waller factor. In describing the scattering of thermal atoms from surfaces, the effect of the attractive well near the surface is usually taken into consideration via the so-called "Beeby correction." 32 For the

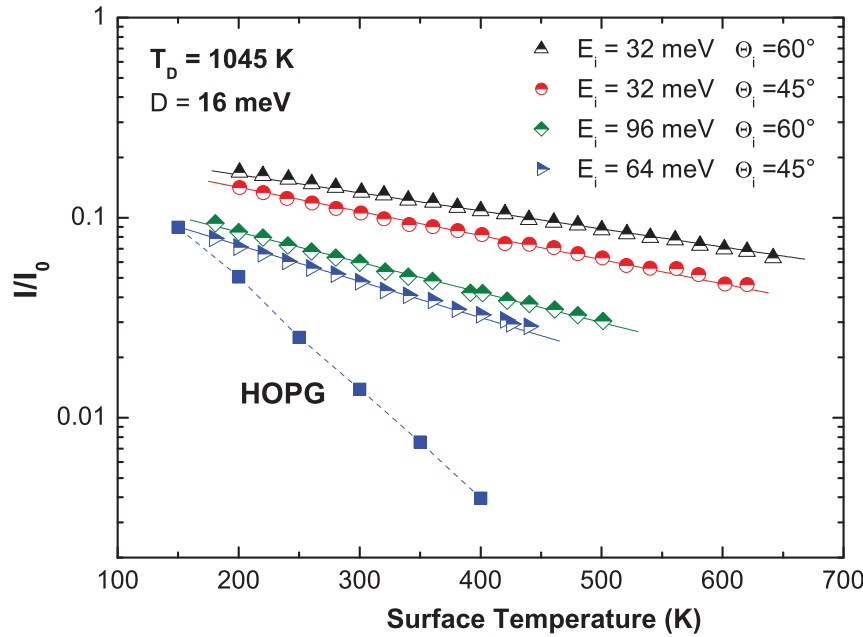

FIG. 3. (Color online) He specular intensity dependence for $\mathrm{MLG} / \mathrm{Ru}(0001)$ as a function of surface temperature, for different incidence angles and energies. The incidence direction was along the [1120] azimuth. Solid lines show the temperature dependence obtained using fits to Eqs. (1) and (2) and the best-fit $D$ value quoted in the text. The corresponding Debye temperature is $1045 \mathrm{~K}$. For comparison, the curve reported in Ref. 26 for HOPG at $E_{i}=63 \mathrm{meV}$ and $\Theta_{i}=45^{\circ}$ is also shown.

specular beam, $W(T)$ can be expressed as a function of the incident beam energy $E_{i}$ and the angle of incidence $\theta_{i}:{ }^{5}$

$$
W(T)=\frac{12 m\left(E_{i} \cos ^{2} \theta_{i}+D\right) T}{M k_{B} \Theta_{D}^{2}},
$$

where $M$ is the mass of a surface atom, $m$ is the mass of the incoming particle, $k_{B}$ is the Boltzmann constant, $\Theta_{D}$ is the surface Debye temperature, and $D$ is the potential well depth. It should be kept in mind that, in some cases, there might be an uncertainty in obtaining the surface Debye temperature from He scattering, since the incoming He atom may interact simultaneously with several surface atoms (due to the long collision time) and then the effective mass $M$ of the surface atom would have to be replaced by the mass of several atoms. In our case, the similar surface structure of supported graphene and graphite avoids this uncertainty.

The solid lines in Fig. 3 are the best fits to the data points using Eq. (2) and $M=12$, corresponding to the mass of a single $\mathrm{C}$ atom. The best-fit parameters are $\Theta_{D}=1045 \pm$ $25 \mathrm{~K}$ and $D=16 \pm 1 \mathrm{meV}$. The attractive potential depth is identical to the one obtained for a previous study on graphite. ${ }^{26}$ Assuming that the effective mass is the same for graphite and graphene, the surface Debye temperature of HOPG is smaller than that of MLG/Ru(0001). The data in Fig. 3 also show that the MLG data follow the dependence on perpendicular momentum transfer predicted by Eq. (2).

These results represent experimental confirmation that the Debye temperature for supported graphene might be significantly higher than the value of $590 \mathrm{~K}$ reported for graphite. ${ }^{26}$ The latter relates to the lattice vibrations along the $c$ axis of graphite, i.e., the perpendicular motion of the outermost layer of graphite, and it is higher than the Debye temperature of most transition metal surfaces, possibly due to the specific phonon density of states of graphite. ${ }^{26}$ 


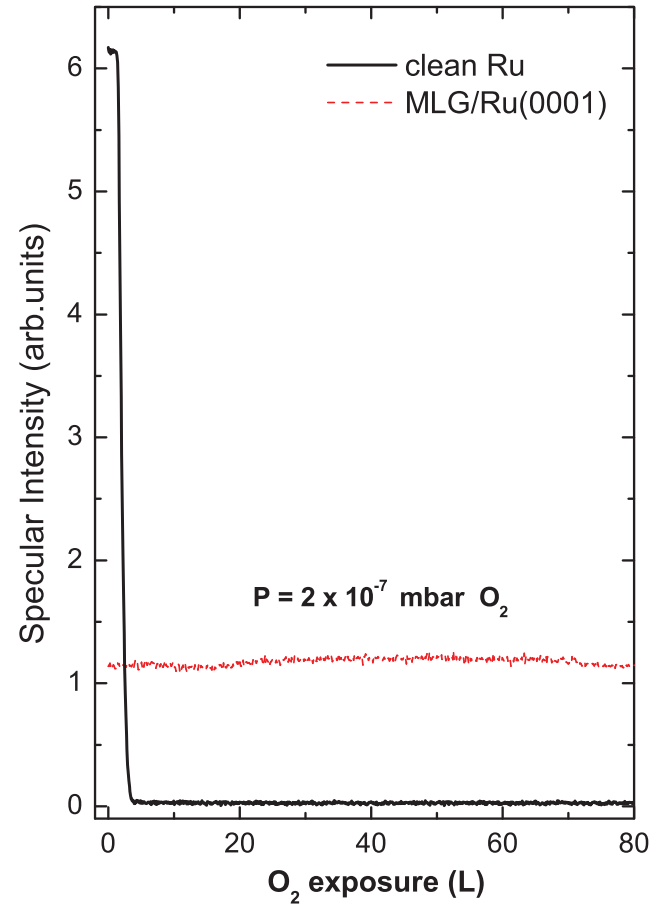

FIG. 4. (Color online) Behavior of the He specular intensity as a function of $\mathrm{O}_{2}$ exposure for the clean $\mathrm{Ru}(0001)$ substrate (black solid curve) and for the MLG/Ru(0001) surface (red dashed curve). The sample temperature is $100 \mathrm{~K}$.

The current results for $\mathrm{MLG} / \mathrm{Ru}(0001)$ can be related to the strong bonding of graphene to $\mathrm{Ru}(0001),{ }^{33}$ which makes it

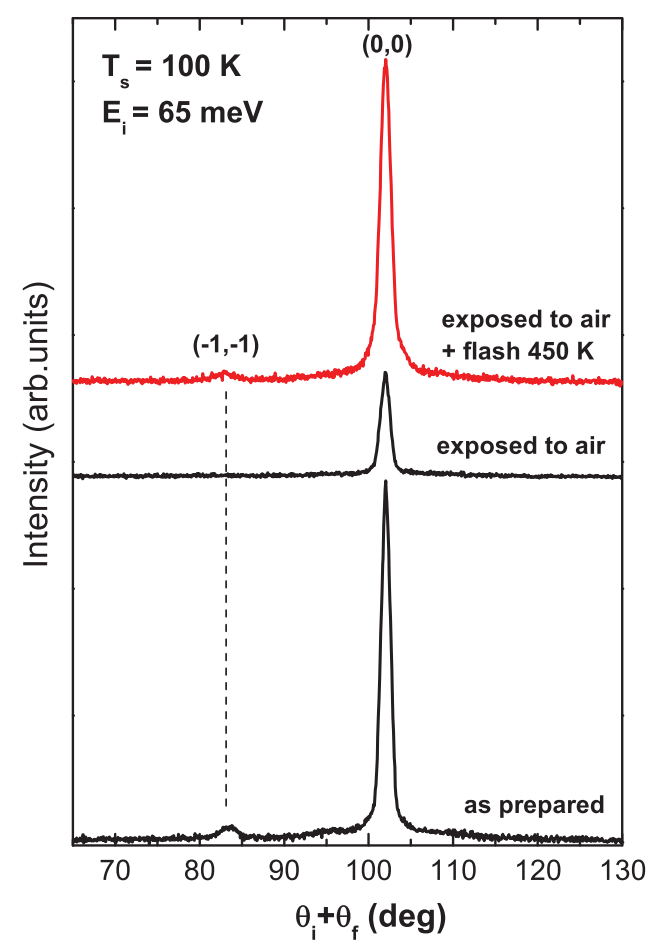

FIG. 5. (Color online) He diffraction in-plane spectra measured for an as-deposited MLG/Ru(0001) surface, successively exposed to air, and finally annealed to $450 \mathrm{~K}$. "harder" than graphite. These findings suggest that the number of low-frequency modes is smaller for graphene/ $\mathrm{Ru}(0001)$ than for graphite.

On the other hand, it is surprising that the Debye temperature of $1287 \mathrm{~K}$ obtained for out-of-plane modes in isolated graphene by phonon Green's function calculations ${ }^{34}$ is close to the value derived from our data, $1045 \mathrm{~K}$. We speculate that it might be due to the occurrence of a weak MLG-Ru bond in the higher regions of the ripples, ${ }^{35}$ which are expected to have a predominant weight in the scattering of the impinging atomic beam. It should be noted that He-atom scattering experiments are not able to provide an estimation of the Debye temperature for planar modes [calculated to be $2000 \mathrm{~K}$ (Ref. 34)], as excitation of transverse modes is not possible within the scattering geometry used in the experiments. It is worth mentioning that the value reported in Ref. 34 is related to zero-point motion in free-standing graphene, while in the present measurements the motion corresponds to thermally activated vibrations.

In addition to the determination of the Debye temperature, the data in Fig. 3 allow us to identify the conditions needed to optimize the specular He reflectivity. From these data, we learn that by combining a large angle of incidence $\left(\Theta_{i} \sim 70^{\circ}\right)$

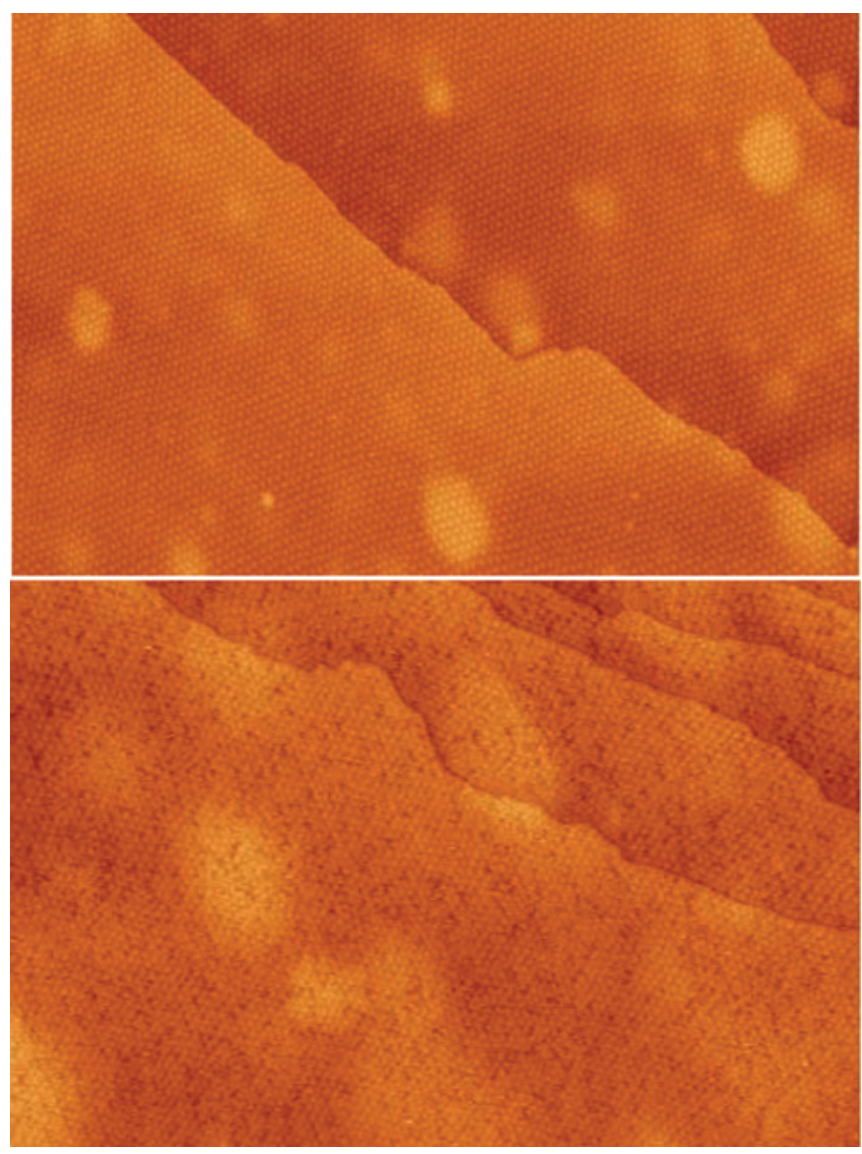

FIG. 6. (Color online) STM images $\left(3000 \times 2000 \AA^{2}\right)$ recorded with a bias voltage of $-1 \mathrm{~V}$ and with a tunneling current of $0.1 \mathrm{nA}$ for the as-deposited MLG on $\mathrm{Ru}(0001)$ before (top) and after air exposure without any thermal treatment (bottom). The ordered bright spots represent the moiré pattern due to the lattice mismatch between graphene and $\mathrm{Ru}(0001)$. 
with an incidence energy close to $E_{i} \sim 10 \mathrm{meV}$, while keeping the surface at $50 \mathrm{~K}$, the specular reflectivity could be increased up to $30 \%$. As a consequence, a SHeAM designed to use these surfaces as mirrors could achieve a signal-to-noise ratio two orders of magnitude larger than current prototypes, which would imply a significant improvement in the lateral resolution achievable.

A practical requirement, however, for using this surface as a focusing mirror in SHeAM is its long-term stability when exposed to contaminants. We have investigated this issue by measuring the surface reactivity in more detail. The results are shown in Fig. 4. Both the bare substrate (black curve) and the MLG/Ru(0001) surface (red curve) were exposed to $\mathrm{O}_{2}$ with the sample kept at $100 \mathrm{~K}$, while monitoring the specular peak intensity $\left(I_{00}\right)$. On the clean surface, a saturated $\mathrm{O} / \mathrm{Ru}(0001)$ layer was formed in a few seconds, upon exposure to just $4 \mathrm{~L}$ of $\mathrm{O}_{2}$. As a consequence, and in spite of its high reflectivity (ca. 35\%), the clean Ru substrate could not be used as a mirror due to its high reactivity.

On the other hand, oxygen adsorption does not occur on MLG/Ru(0001), as indicated by the nearly constant behavior of $I_{00}$ during $\mathrm{O}_{2}$ exposure. These findings demonstrate that $\mathrm{MLG} / \mathrm{Ru}(0001)$ is inert even at $100 \mathrm{~K}$. Previously, the reactivity of MLG toward oxygen has been addressed by studies performed only at room temperature, ${ }^{36,37}$ at which the physisorption of molecules is usually quite reduced with respect to the case of $100 \mathrm{~K}$. In addition, we have exposed the sample to air for a few minutes and transferred it back to the UHV chamber. The results, presented in Fig. 5, show that a significant He-beam reflectivity is still obtained from MLG, even without performing any cleaning procedure, which is a very surprising result. We have also observed that the reflectivity of the as-deposited sample is fully recovered (within our experimental error of 5\%) after flash annealing the sample to $450 \mathrm{~K}$, as illustrated by the red curve in Fig. 5. STM experiments carried out on the air-exposed sample (without any thermal treatment) show that the MLG/Ru(0001) film remains almost unaffected by the interaction with air (Fig. 6). In particular, after reintroduction of the sample into the UHV system, the same $(12 \times 12)$ Moiré pattern ${ }^{16,17,19}$ as in the as-deposited MLG sample was observed.

\section{CONCLUSIONS}

We have demonstrated that MLG/Ru(0001) is an excellent mirror for He atoms, with an absolute specular reflectivity of $20 \%$. This surface is thermally stable up to $1150 \mathrm{~K}$ and its $\mathrm{He}$ reflectivity remains almost unchanged even after exposure to air. On the basis of such findings, a significant improvement in the lateral resolution achievable is expected for the prototypes of the SHeAM designed to use MLG surfaces as mirrors. The MLG/Ru(0001) surface works as a mirror not only for $\mathrm{He}$ but also for $\mathrm{H}_{2}$ beams, for which an extremely high reflectivity of $11 \%$ has been obtained. The Debye temperature for $\mathrm{MLG} / \mathrm{Ru}(0001)$ has been determined to be $1045 \mathrm{~K}$, a value significantly higher than the one reported for graphite ${ }^{26}$ $(590 \mathrm{~K})$, and very close to the value of $1287 \mathrm{~K}$ calculated for self-suspended graphene. ${ }^{34}$

\section{ACKNOWLEDGMENTS}

This work was supported by the Ministerio de Educación y Ciencia through the program CONSOLIDER-INGENIO on Molecular Nanoscience (Project No. CSD 2007-00010), Project No. FIS2010-18847, and a Juan de la Cierva grant (A.P.), and by Comunidad de Madrid through the program NANOBIOMAGNET. *daniel.farias@uam.es

${ }^{1}$ B. Holst and W. Allison, Nature (London) 390, 244 (1997).

${ }^{2}$ M. Koch, S. Rehbein, G. Schmahl, T. Reisinger, G. Bracco, W. E. Ernst, and B. Holst, J. Microsc. 229, 1 (2008).

${ }^{3}$ T. Reisinger, S. Eder, M. M. Greve, H. I. Smith, and B. Holst, Microelectron. Eng. 87, 1011 (2010).

${ }^{4}$ T. Reisinger and B. Holst, J. Vac. Sci. Technol. B 26, 2374 (2008).

${ }^{5}$ D. Farías and K. H. Rieder, Rep. Prog. Phys. 61, 1575 (1998).

${ }^{6}$ R. Ramachandra, B. Griffin, and D. Joy, Ultramicroscopy 109, 748 (2009).

${ }^{7}$ D. C. Bell, Microsc. Microanal. 15, 147 (2009).

${ }^{8}$ R. B. Doak, R. E. Grisenti, S. Rehbein, G. Schmahl, J. P. Toennies, and C. Woll, Phys. Rev. Lett. 83, 4229 (1999).

${ }^{9}$ T. Reisinger, G. Bracco, S. Rehbein, G. Schmahl, W. E. Ernst, and B. Holst, J. Phys. Chem. A 111, 12620 (2007).

${ }^{10}$ D. A. MacLaren, W. Allison, and B. Holst, Rev. Sci. Instrum. 71, 2625 (2000).

${ }^{11}$ D. Barredo, F. Calleja, A. E. Weeks, P. Nieto, J. J. Hinarejos, G. Laurent, A. L. Vázquez de Parga, D. A. MacLaren, D. Farías, W. Allison, and R. Miranda, Surf. Sci. 601, 24 (2007).

${ }^{12}$ K. Fladischer, H. Reingruber, T. Reisinger, V. Mayrhofer, W. E. Ernst, A. E. Ross, D. A. MacLaren, W. Allison, D. Litwin, J. Galas, S. Sitarek, P. Nieto, D. Barredo, D. Farías, R. Miranda, B. Surma,
A. Miros, B. Piatkowski, E. Søndergård, and B. Holst, New J. Phys. 12, 033018 (2010)

${ }^{13}$ D. Barredo, F. Calleja, P. Nieto, J. J. Hinarejos, G. Laurent, A. L. Vázquez de Parga, D. Farías, and R. Miranda, Adv. Mater. 20, 3492 (2008).

${ }^{14}$ D. Barredo, G. Laurent, F. Calleja, P. Nieto, J. J. Hinarejos, A. L. Vázquez de Parga, D. Farías, and R. Miranda, Appl. Phys. Lett. 96, 081901 (2010).

${ }^{15}$ S. Marchini, S. Gunther, and J. Wintterlin, Phys. Rev. B 76, 075429 (2007).

${ }^{16}$ A. L. Vázquez de Parga, F. Calleja, B. Borca, M. C. G. Passeggi, J. J. Hinarejos, F. Guinea, and R. Miranda, Phys. Rev. Lett. 100, 056807 (2008).

${ }^{17}$ P. W. Sutter, J. I. Flege, and E. A. Sutter, Nature Mater. 7, 406 (2008).

${ }^{18}$ I. Pletikosic, M. Kralj, P. Pervan, R. Brako, J. Coraux, A. T. N'Diaye, C. Busse, and T. Michely, Phys. Rev. Lett. 102, 056808 (2009).

${ }^{19}$ Y. Pan, H. Zhang, D. Shi, J. Sun, S. Du, F. Liu, and H.-J. Gao, Adv. Mater. 21, 2777 (2009).

${ }^{20}$ E. Sutter, P. Albrecht, and P. Sutter, Appl. Phys. Lett. 95, 133109 (2009).

${ }^{21}$ P. W. Sutter, P. M. Albrecht, and E. A. Sutter, Appl. Phys. Lett. 97, 213101 (2010) 
${ }^{22}$ A. Bostwick, J. McChesney, T. Ohta, E. Rotenberg, T. Seyller, and K. Horn, Prog. Surf. Sci. 84, 380 (2009).

${ }^{23}$ N. W. Ashcroft and N. D. Mermin, Solid State Physics (HoltSaunders, New York, 1976).

${ }^{24}$ A. C. Levi and H. Suhl, Surf. Sci. 88, 221 (1979).

${ }^{25}$ A. C. Levi, J. Phys.: Condens. Matter 21, 405004 (2009).

${ }^{26}$ J. P. Oh, T. Kondo, D. Hatake, and J. Nakamura, Surf. Sci. 603, 895 (2009).

${ }^{27}$ P. Nieto, D. Barredo, D. Farías, and R. Miranda J. Phys. Chem. A 115, 7283 (2011).

${ }^{28}$ H. Zhang, Q. Fu, Y. Cui, D. Tan, and X. Bao, J. Phys. Chem. C 113, 8296 (2009).

${ }^{29}$ B. Borca, S. Barja, M. Garnica, M. Minniti, A. Politano, J. M. Rodríguez-García, J. J. Hinarejos, D. Farías, A. L.
Vázquez de Parga, and R. Miranda, New J. Phys. 12, 075429 (2010).

${ }^{30}$ G. S. McCarty, Nano Lett. 4, 1391 (2004).

${ }^{31}$ B. Gumhalter, Phys. Rep. 351, 1 (2001).

${ }^{32}$ J. L. Beeby, J. Phys. C 4, L359 (1971).

${ }^{33}$ P. W. Sutter, M. S. Hybertsen, J. T. Sadowski, and E. Sutter, Nano Lett. 9, 2654 (2009).

${ }^{34}$ V. K. Tewary and B. Yang, Phys. Rev. B 79, 125416 (2009).

${ }^{35}$ B. Wang, S. Günther, J. Wintterlin, and M.-L. Bocquet, New J. Phys. 12, 043041 (2010).

${ }^{36}$ B. Borca, F. Calleja, J. J. Hinarejos, A. L. Vázquez de Parga, and R. Miranda, J. Phys.: Condens. Matter 21, 134002 (2009).

${ }^{37}$ Y. S. Dedkov, M. Fonin, U. Rdiger, and C. Laubschat, Appl. Phys. Lett. 93, 22509 (2008). 